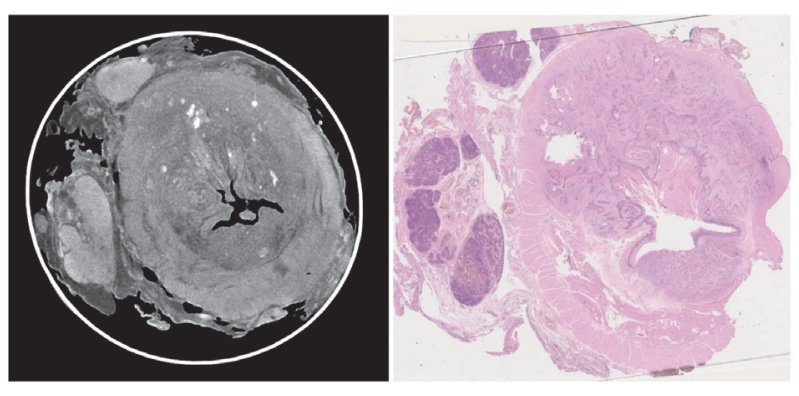

Abstract P239 Figure 1 CT slice showing oesophageal tumour with matched histology section

We reconstructed the images using MATLAB $^{\circledR}$ software. Specimens were returned for clinical histopathological assessment allowing correlation between H\&E slides and CT images.

Results We have performed 25 scans on 10 oesophagectomy samples and correlated them with histology

Scans of samples in formalin failed to show adequate contrast between oesophageal layers to enable tumour visualisation and staging. Infiltrating the tissue with ethanol led to much better image contrast.

We could easily identify mucosa, submucosa and both layers of muscle in reconstructed CT images. We also identified tumour infiltration through tissue layers and destruction of normal oesophageal morphology (figure 1). This was confirmed histologically and could be recognised by radiologists blinded to pathological staging

This is the first time that XPCI has been used to image human oesophageal tissue. We have demonstrated the feasibility of the technique and the possibility of obtaining high resolution images which mimic histology with the extra benefit of demonstrating three dimensional structure.

\section{P240 ACCURACY OF CLINICAL STAGING FOR T2NO OESOPHAGEAL CANCER: SYSTEMATIC REVIEW AND META-ANALYSIS}

${ }^{1}$ Paul Wolfson*, ${ }^{1}$ Alexander Ho, ${ }^{2}$ Paul Bassett, ${ }^{1}$ Rehan Haidry, ${ }^{1}$ Laurence Lovat,
${ }^{1}$ Sarmed Sami. ${ }^{1}$ University College London, London, UK; ${ }^{2}$ Stats Consultancy Ltd, Amersham,
UK

10.1136/gutjnl-2020-bsgcampus.314

Background and Aims Oesophageal cancer is the 7th most common cause of cancer worldwide and is the 6th most common cause of overall cancer mortality. Clinical staging utilises multiple imaging modalities and is the most appropriate guide for treatment and prognostication. T2N0 oesophageal cancer is a treatment threshold for neo-adjuvant therapy, but data on the accuracy of current clinical staging tests for identifying this disease subgroup are conflicting.

Methods We performed a meta-analysis of all primary studies evaluating the accuracy of clinical staging (index test) compared to histopathological staging of oesophagectomy specimens (reference standard) in T2N0 oesophageal cancer. Clinical staging used a combination of PET, EUS, and CT imaging modalities. Patients were excluded if they had neoadjuvant therapy to allow direct comparison between clinical and pathological staging. Databases searched included: Ovid

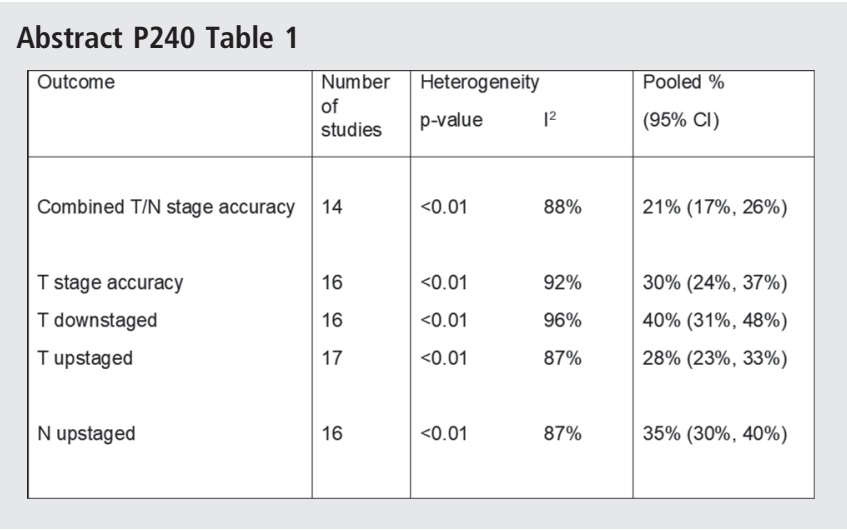

MEDLINE, Ovid Embase and The Cochrane Library up to September 2019.

The primary outcome was diagnostic accuracy of combined $\mathrm{T}$ and $\mathrm{N}$ clinical staging. Secondary outcomes were accuracy of $\mathrm{T}$ stage; percentage $\mathrm{T}$ downstaged; percentage $\mathrm{T}$ upstaged and percentage $\mathrm{N}$ upstaged.

We evaluated several sources of heterogeneity a priori including: publication date (before and after Jan 2015), date of first recruitment (before and after 2000), number of centre (single vs multicentre), sample size $(<100$ vs $\geq 100$ ), geographical location (USA, Europe or Asia) and main histological subtype (adenocarcinoma or squamous cell carcinoma).

Results After removing duplicates, the search strategy identified 1,199 studies which were all screened by title and abstract. Eighteen studies met the inclusion criteria containing 5,115 patients. The combined T\&N staging accuracy was 21\% (95\% confidence interval (CI), 17-26); T stage accuracy was 30\% (95\%CI, 24-37); percentage of patients with $\mathrm{T}$ down-staging was $40 \%$ (95\% CI, 31-48); percentage of patients with $\mathrm{T}$ upstaging was $28 \%(95 \% \mathrm{CI}, 23-33)$ and the percentage of patients with $\mathrm{N}$ upstaging was $35 \%$ (95\% CI, 30-40)(table 1).

Sources of heterogeneity in accuracy of T\&N staging included: number of centres (single centre $=15 \%$ vs multicentre studies $=27 \% ; \quad \mathrm{p}=0.01) ;$ sample size $\quad(\mathrm{n} \leq 100$ patients $=15 \%$ vs $\mathrm{n} \geq 100$ patients $=27 \% ; \mathrm{p}=0.01$ ); study region (USA $=19 \%$ vs Asia $=19 \%$ vs Europe $=38 \%$; $<0.01$ ).

Conclusion Staging for T2N0 oesophageal cancer remains inaccurate with a significant proportion of patients having their disease downstaged (therefore, could have been potentially offered endotherapy instead of oesophagectomy) as well as upstaged (therefore, they could have been potentially offered neo-adjuvant therapy). These findings were largely unchanged over the past two decades suggesting that there is an urgent need for more accurate staging tests for this subgroup of patients.

\section{Gastroduodenal}

\section{P241 NO ACUTE CLINICALLY-SIGNIFICANT EVENTS DUE TO HYPOPHOSPHATAEMIA POST-FERRIC CARBOXYMALTOSE THERAPY: AN AUDIT}

Nicole Cianci*, Zin Tun, Khin Thein, AUF Jawhari. Nottingham University Hospitals Nhs Trust, Nottingham, UK

10.1136/gutjnl-2020-bsgcampus.315 